First publ. in: Surface Science 98 (1980), 1-3, p.68

\title{
THE DIMPLE CRYSTAL - A CHARGE-INDUCED INSTABILITY OF THE HELIUM SURFACE
}

\author{
P. LEDERER and M. WANNER \\ Fachbereich Physik der Technischen Universität München, D-8046 Garching, West Germany
}

Two-dimensional systems of charge carriers on a liquid substrate have been predicted to show an instability resulting from a softening of the excitation spectrum of the liquid surface. We have investigated two different types of such Coulomb systems on exceptionally pure substrates: (i) quasi-free electrons above the free surface of liquid ${ }^{4} \mathrm{He}$; (ii) positive and negative ions at the interface of phase-separated ${ }^{3} \mathrm{He}-{ }^{4} \mathrm{He}$ mixtures [1]. For the latter, the suggested softening of interfacial excitations due to their interaction with ions in an external electric field has been verified experimentally [2].

Moreover, at a critical field $E_{\mathrm{c}}$ a phase transition has been observed to a state where the charge carriers are no longer distributed homogeneously throughout the interface, but in a periodic array of groups of typically $10^{6}$ ions, giving rise to a strikingly regular pattem of interfacial indentations, the "dimple crystal". A similar transition was found also for electrons at the free helium surface [3]. The structure of the dimple lattice is in both cases hexagonal.

One can consider different types of dimple crystals: As long as the depth of the dimples is small and the electrostatic potential along the surface is constant, the dimple lattice represents a charge density wave modulating the Coulomb gas or for low enough temperature - the Wigner crystal formed by the individual charge carriers. When the indentations are deep, on the other hand, all the charges are concentrated in the dimple minima and cannot move from one indentation to the other. Since the charge in each dimple is therefore fixed, the dimple lattice can in this case be regarded as a kind of macroscopic two-dimensional Wigner crystal, modified by additional attractive forces between the dimples due to the deformation of the surface.

It should be possible to distinguish between these two types of lattices because their excitation spectra are expected to be different.

\section{References}

[1] P. Leidcrer, M. Wanner and W. Schoepe, J. Physique C6 (1978) 1327.

[2] M. Wanner and P. Leiderer, Phys. Rev. Letters 42 (1979) 315.

[3] P. Leiderer and M. Wanner, to be published. 